\title{
QUALIDADE DAS SEMENTES DE SOJA DAS FRAÇÕES E DO REPASSE BENEFICIADAS NA MESA DE GRAVIDADE
}

\author{
Alcione Lorenset ${ }^{1}$, Aline Klug Radke ${ }^{2}$, Jean Carlo Possenti ${ }^{3}$, Francisco Amaral Villela ${ }^{2}$ \\ ${ }^{1}$ C. Vale - Cooperativa Agroindustrial, DEAGI - Produção de Sementes, Abelardo Luz, SC. ${ }^{2}$ Universidade Federal de \\ Pelotas - UFPel, Faculdade de Agronomia Eliseu Maciel - FAEM, Pelotas, RS. ${ }^{3}$ Universidade Tecnológica Federal do \\ Paraná, Campus de Dois Vizinhos, PR. E-mail: alinekradke@hotmail.com
}

\section{RESUMO}

O presente trabalho teve por objetivo verificar a qualidade física e fisiológica de sementes de soja após o beneficiamento na mesa de gravidade e determinar as características da fração do repasse após passar novamente pela mesa de gravidade. Foi utilizado um lote de sementes, cultivar BMX Potencia RR, proveniente de campo de Abelardo Luz. Inicialmente, as sementes foram beneficiadas em máquina de ar e peneiras, separador espiral, classificador, mesa de gravidade. Foram coletadas amostras, dividindo o eixo terminal da descarga em 10 partes iguais de largura $10 \mathrm{~cm}$ cada, constituindo os tratamentos denominados de frações 1 a 10, sendo 1 a parte mais alta e 10 a parte mais baixa da zona de descarga. Após a coleta, foram separadas em partes medianas da mesa (frações 5 a 7) formando a fração a ser repassada pela mesa dividindo o eixo de descarga das sementes em 10 partes iguais de $10 \mathrm{~cm}$ cada, constituindo os tratamentos denominados de frações 11 a 20. As sementes foram submetidas às seguintes avaliações: germinação, envelhecimento acelerado, tetrazólio, peso de mil sementes e hipoclorito de sódio. As frações de maior qualidade física e fisiológica representam 80 \% do lote de sementes ao serem beneficiadas na mesa de gravidade. A qualidade física e fisiológica da fração repasse na mesa de gravidade representa em média 10 $\%$ do lote e permite um aproveitamento de até $80 \%$.

Palavras-chave: Glycine max,; mesa densimétrica; germinação; vigor.

\section{QUALITY OF THE SOYBEAN SEEDS OF FRACTIONS AND REPRODUCTION BENEFITED ON THE GRAVITY TABLE}

\begin{abstract}
The objective of the present work was to verify the physical and physiological quality of soybean seeds after the treatment at the gravity table and to determine the characteristics of the transfer fraction after passing through the gravity table again. A batch of seeds, cultivar BMX Potencia RR, from the Abelardo Luz field was used. Initially, the seeds were benefited in air machine and sieves, spiral separator, classifier, gravity table. Samples were collected dividing the terminal axis of the discharge into 10 equal parts of width $10 \mathrm{~cm}$ each, which were the treatments denominated fractions 1 to 10 , with 1 being the highest part and 10 being the lowest part of the discharge zone. After the collection, the medial parts of the table (fractions 5 to 7) were separated that formed the fraction to be transferred by the table dividing the axis of discharge of the seeds in 10 equal parts of $10 \mathrm{~cm}$ each, constituting the denominated treatments of fractions 11 to 20. The seeds were submitted to the following evaluations: germination, accelerated aging, tetrazolium, thousand seed weight and mechanical damage.. The higher physical and physiological fractions represent $80 \%$ of the seed lot when benefited at the gravity table. The physical and physiological quality of the fraction passed on the gravity table represents on average $10 \%$ of the lot and allows a recovery of up to 80 $\%$.
\end{abstract}

Key-words: Glycine max; densimetric table; germination; vigor. 


\section{INTRODUÇÃO}

A produção de sementes de qualidade boa qualidade constitui o principal elemento de marketing das empresas que configuram em um mercado de alta competitividade, sendo para isso imprescindível garantir a integração entre as etapas de produção, beneficiamento, análise e comercialização. Segundo Tunes et al. (2011) a qualidade das sementes pode ser caracterizada pela germinação e pelo vigor, o qual pode ser definido como a somatória de atributos que conferem, à semente, o potencial de germinar e emergir refletindo diretamente no desenvolvimento da cultura gerando plantas de elevado vigor.

O beneficiamento de sementes é parte essencial da tecnologia envolvida na produção de sementes de alta qualidade e tem o objetivo de separar dos lotes de sementes, os materiais indesejáveis como impurezas, sementes de plantas invasoras, sementes imaturas, mal formadas e deterioradas, e as atacadas por fungos e insetos (DESCHAMPS, 2005), visando o melhoramento das características físicas de um lote de sementes (PESKE; BAUDET, 2003). Neste processo, a mesa de gravidade pode aprimorar a qualidade sanitária de lotes de sementes, pela remoção de sementes infectadas e atacadas por insetos, conforme verificado por Buitrago et al. (1991), em sementes de feijão e por Bicca (1996), em sementes de arroz.

Entretanto, nem todos os lotes seguem uma mesma sequência, de forma que as operações neste processo são realizadas em função da espécie, da cultivar e das características físicas e das impurezas presentes (FERREIRA; SÁ, 2010). Segundo Marcos Filho (2013) o embrião da semente de soja é protegido por um tegumento relativamente frágil, o eixo embrionário é superficial e suscetível a injúrias mecânicas provocadas por outros agentes externos demonstrando que a semente pode sofrer injúrias mecânicas durante as etapas do beneficiamento. Frente a isso é importante conhecer se existe ou não variabilidade espacial da qualidade fisiológica das sementes, durante seu beneficiamento.

Assim, este estudo teve por objetivo avaliar a qualidade física e fisiológica de lotes de sementes de soja após o beneficiamento na mesa de gravidade e determinar as características físicas e fisiológicas da fração do repasse após passarem novamente pela mesa de gravidade.

\section{MATERIAL E MÉTODOS}

O trabalho foi conduzido na unidade de beneficiamento de sementes da C.Vale Cooperativa Agroindustrial de Abelardo Luz - SC e os testes de germinação e vigor foram realizados no laboratório de sementes (LASP) da mesma cooperativa em Palotina -PR.

Foi utilizado um lote de sementes de soja, cultivar BMX Potencia RR, proveniente de campo de produção em Abelardo Luz - SC. As sementes foram colhidas, pré-limpas, secas e armazenadas, com umidade de $13,2 \%$, em big bag até a data de beneficiamento em agosto.

As sementes foram beneficiadas em máquina de ar e peneiras, separador espiral, classificador e mesa de gravidade (retangular da marca Rota, modelo RT 80, com capacidade operacional de 3,2 $\mathrm{t} / \mathrm{h}$ ).

Para a coleta das amostras das sementes - eixo terminal de descarga da mesa de gravidade foi dividido em 10 partes iguais de largura $10 \mathrm{~cm}$ cada uma, que constituíram os tratamentos denominados de frações 1 a 10, sendo 1 a parte mais alta e 10 a parte mais baixa da zona de descarga. As frações medianas da mesa (frações 5 a 7) formaram a fração a ser repassada na mesa de gravidade, dividindo novamente o eixo terminal de descarga das sementes em 10 partes iguais de largura $10 \mathrm{~cm}$ cada uma, constituindo os tratamentos denominados de frações 11 a 20.

Inicialmente, foram coletadas amostras nas frações de 1 a 10, sendo coletadas em cada ponto em intervalos regulares de 10 minutos, utilizando-se recipientes com $10 \mathrm{~cm}$ de largura e capacidade de $1 \mathrm{~kg}$, empregando quatro repetições. Posteriormente, coletaram-se amostras das frações de 11 a 20, seguindo procedimento similar ao da amostragem das 10 primeiras frações.

As análises físicas e fisiológicas foram as seguintes:

Peso de mil sementes: foram utilizadas oito subamostras de 100 sementes para a determinação do peso de mil sementes, conforme procedimento das Regras para Análise de Sementes - RAS (BRASIL, 2009)

Hipoclorito de sódio: foram utilizadas duas amostras de 100 sementes, as sementes foram mergulhadas em solução de hipoclorito de sódio a 5,25 \% durante 10 minutos, após as sementes foram espalhadas em papel toalha para avaliação e contagem das sementes entumecidas, 
conforme metodologia descrita por Krzyzanowski et al. (2004).

Germinação: conduzido com quatro subamostras de 50 sementes, semeados em papel Germitest umedecido com água na proporção 2,5 vezes a massa do substrato seco, permanecendo em germinador a $25{ }^{\circ} \mathrm{C}$. As avaliações foram realizadas aos cinco e catorze dias após a semeadura. Os resultados foram expressos em porcentagem média de plântulas normais (BRASIL, 2009).

Envelhecimento acelerado: foram utilizadas 200 sementes (quatro subamostras de 50 sementes) para cada amostra. O teste foi conduzido em caixas gerbox, contendo $40 \mathrm{~mL}$ de água destilada e uma camada uniforme de sementes dispostas sobre a tela interna, mantidas em incubação a $41{ }^{\circ} \mathrm{C}$, por 24 horas. Após o período de envelhecimento, as sementes foram submetidas ao teste de germinação, sendo a avaliação realizada no quinto dia após a semeadura (MARCOS FILHO, 1999).

Teste de tetrazólio: foram utilizadas 400 sementes divididas em oito repetições de 50, seguindo os critérios das Regras para Análise de Sementes (BRASIL, 2009).

O delineamento experimental utilizado foi inteiramente casualizado, com 20 tratamentos (20 frações) e quatro repetições. As médias dos tratamentos foram comparadas pelo teste de Scott-Knott, em nível de $5 \%$ de probabilidade. As análises dos dados foram efetuadas separadamente para cada atributo de qualidade, tanto na primeira passagem das sementes na mesa de gravidade como para o repasse, com o auxílio do software computacional ASSISTAT, Versão 7.6 Beta.

\section{RESULTADOS E DISCUSSÃO}

O lote de sementes utilizado inicialmente possuía $18.800 \mathrm{~kg}$, sendo que após a primeira passagem pela mesa de gravidade, as frações 5 a 7 atingiram a quantidade de $1.920 \mathrm{~kg}(10 \%)$, o descarte foi de $510 \mathrm{~kg}(2,7 \%)$ e o aproveitamento de sementes alcançou $13.450 \mathrm{~kg}$ (71 \%). O descarte na máquina de ar e peneiras, separador de espiral e padronizador atingiu 16,3\%. No repasse de $1.920 \mathrm{~kg}$ provenientes das frações que retornaram para a mesa de gravidade, o aproveitamento foi de $1550 \mathrm{~kg}$ de semente $(8,2 \%$ em relação ao peso inicial), totalizando um aproveitamento de $79,2 \%$. A porção correspondente às frações de provenientes das etapas 5 a 7 totalizou $310 \mathrm{~kg}$ e o descarte foi de $60 \mathrm{~kg}$, significando um descarte total na mesa de gravidade de 4,6 \%, ao não aproveitar também o material das frações 5 a 7 obtidos após o repasse.

Quanto à qualidade física (Tabela 1) peso de mil sementes (PMS) e dano mecânico (DM) - as sementes de maior qualidade concentraram-se nas posições superiores da zona de descarga da mesa de gravidade, evidenciando um aumento progressivo de qualidade da posição mais baixa (10) para a posição mais alta (1) do eixo terminal de descarga. 0 repasse, caracterizado pelos tratamentos de 11 a 20, apresentou maior estratificação quanto ao peso de 1000 sementes, sendo as de maior peso situadas até $40 \mathrm{~cm}$ a partir da parte alta.

A diferença de peso de 1000 sementes entre as frações 1 e 10 foi de $19 \mathrm{~g}$, correspondente a $14 \%$ e entre as frações 11 e 20 alcançou $7,7 \%$. As frações compreendidas na faixa de 7 a 10 mostraram similaridade com a faixa das frações de 17 a 20.

Com base nos resultados obtidos pode-se inferir que a fração 5 apresentou resultados similares às frações11 a 14, provenientes do repasse que poderiam ser aproveitadas, pois apresentam peso de 1000 sementes $4,4 \%$ menor do que a fração 1 . As frações 6 e 16 não diferiram quanto ao peso de 1000 sementes, embora a diferença em relação à fração 1 seja de $6 \%$.

No que se refere à incidência de dano mecânico, as frações $9,10,18,19$ e 20 foram significativamente inferiores em relação às demais frações, com incidências variáveis de 5,75 a $8,0 \%$.

A concentração das sementes danificadas nas frações localizadas na parte baixa da mesa de gravidade e com possibilidade de remoção, pelo descarte dessas frações, poderá consequentemente aumentar a qualidade física do lote, conforme constatado por Gaul et al. (1986) e Silva Filho (1994). 
Tabela 1. Qualidade física - peso de 1000 sementes (PMS) e dano mecânico (DM) de sementes de soja obtidas em 10 posições ( 1 a 10) do eixo terminal de descarga e 10 posições (11 a 20) do eixo terminal de descarga no repasse na mesa de gravidade.

\begin{tabular}{ccc}
\hline $\begin{array}{c}\text { Posição na mesa } \\
\text { (Tratamento) }\end{array}$ & PMS & DM \\
\hline $\mathbf{0 1}$ (Parte alta) & $132.43750 \mathrm{a}$ & $3,00 \mathrm{a}$ \\
$\mathbf{0 2}$ & $131.36000 \mathrm{a}$ & $3,00 \mathrm{a}$ \\
$\mathbf{0 3}$ & $130.96000 \mathrm{a}$ & $3,50 \mathrm{a}$ \\
$\mathbf{0 4}$ & $129.57750 \mathrm{~b}$ & $3,00 \mathrm{a}$ \\
$\mathbf{0 5}$ & $126.65250 \mathrm{c}$ & $3,75 \mathrm{a}$ \\
$\mathbf{0 6}$ & $124.17000 \mathrm{~d}$ & $4,00 \mathrm{a}$ \\
$\mathbf{0 7}$ & $122.49750 \mathrm{e}$ & $3,25 \mathrm{a}$ \\
$\mathbf{0 8}$ & $119.05500 \mathrm{~g}$ & $4,50 \mathrm{a}$ \\
$\mathbf{0 9}$ & $114.77750 \mathrm{~h}$ & $6,50 \mathrm{~b}$ \\
$\mathbf{1 0}$ (Parte baixa) & $113.46250 \mathrm{~h}$ & $8,00 \mathrm{c}$ \\
$\mathbf{1 1}$ (Parte alta) & $128.47250 \mathrm{c}$ & $3,50 \mathrm{a}$ \\
$\mathbf{1 2}$ & $128.17750 \mathrm{c}$ & $3,75 \mathrm{a}$ \\
$\mathbf{1 3}$ & $127.59250 \mathrm{c}$ & $3,50 \mathrm{a}$ \\
$\mathbf{1 4}$ & $126.47000 \mathrm{c}$ & $3,75 \mathrm{a}$ \\
$\mathbf{1 5}$ & $125.84750 \mathrm{~d}$ & $4,00 \mathrm{a}$ \\
$\mathbf{1 6}$ & $124.34750 \mathrm{~d}$ & $4,50 \mathrm{a}$ \\
$\mathbf{1 7}$ & $123.22250 \mathrm{e}$ & $3,50 \mathrm{a}$ \\
$\mathbf{1 8}$ & $120.83750 \mathrm{f}$ & $5,75 \mathrm{~b}$ \\
$\mathbf{1 9}$ & $120.05500 \mathrm{f}$ & $6,50 \mathrm{~b}$ \\
$\mathbf{1 9}$ (Parte baixa) & $118.17750 \mathrm{~g}$ & $7,25 \mathrm{c}$ \\
\hline CV (\%) & 1,00 & 19,5 \\
\hline
\end{tabular}

Médias seguidas pela mesma letra não diferem entre si.pelo teste de Scott-Knott em nível de 5 \% de probabilidade

A Tabela 2 apresenta os resultados dos testes de germinação, envelhecimento acelerado, tetrazólio e viabilidade, parâmetros utilizados para determinar a qualidade fisiológica das sementes de soja retiradas nas 10 posições do eixo terminal de descarga da mesa de gravidade e nas 10 posições no repasse.

Observa-se aumento da qualidade fisiológica das sementes no sentido ascendente do eixo terminal de descarga da mesa de gravidade ficando evidentes estas diferenças nas sementes, ou seja, o aumento na qualidade final do lote de sementes se deu, pois houve repasse das sementes na mesa de gravidade. Estes resultados vão de encontro ao estudo de Juvino et al. (2014) que trabalhando com sementes de soja observou que existe diferenças na qualidade fisiológica das sementes entre as etapas do beneficiamento.
A germinação foi superior nas frações da faixa de 1 a 5 e na fração 11, intermediária nas frações nas faixas de 6 a 8 e de 12 a 17 e inferior nas faixas de 9 a 19 e de 18 a 20, sendo que as sementes de germinação inferior não atingiram $80 \%$, percentual mínimo exigido na comercialização.

A viabilidade das sementes determinada pelo teste de tetrazólio também permitiu estratificar as frações em três níveis, sendo superior nas frações das faixas de 1 a 8 e de 11 a 15 , médio na fração 9 e na faixa de 16 a 18 e inferior na frações 10, 19 e 20.

$\mathrm{O}$ vigor de sementes, avaliado pelo teste de tetrazólio, possibilitou a separação das frações em três níveis, com superioridade nas frações da faixa de 1 a 4 e na fração 11 , vigor intermediário nas faixas de 5 a 6 e de 12 a 15 e inferioridade nas faixas de 7 a 10 e de 15 a 20 . 
Tabela 2. Qualidade fisiológica - germinação (G), tetrazólio-viabilidade (TZ), tetrazólio-vigor (TZV) e envelhecimento acelerado (EA) de sementes de soja obtidas em 10 posições ( 1 a 10) do eixo terminal de descarga e 10 posições (11 a 20) no repasse da mesa de gravidade.

\begin{tabular}{ccccc}
\hline Posição da mesa & G (\%) & TZ (\%) & TZV (\%) & EA (\%) \\
\hline $\mathbf{0 1}$ (Parte alta) & $92 \mathrm{a}$ & $86 \mathrm{a}$ & $77 \mathrm{a}$ & $86 \mathrm{a}$ \\
$\mathbf{0 2}$ & $92 \mathrm{a}$ & $87 \mathrm{a}$ & $73 \mathrm{a}$ & $87 \mathrm{a}$ \\
$\mathbf{0 3}$ & $91 \mathrm{a}$ & $87 \mathrm{a}$ & $76 \mathrm{a}$ & $84 \mathrm{a}$ \\
$\mathbf{0 4}$ & $90 \mathrm{a}$ & $89 \mathrm{a}$ & $75 \mathrm{a}$ & $84 \mathrm{a}$ \\
$\mathbf{0 5}$ & $90 \mathrm{a}$ & $87 \mathrm{a}$ & $68 \mathrm{~b}$ & $85 \mathrm{a}$ \\
$\mathbf{0 6}$ & $88 \mathrm{~b}$ & $84 \mathrm{a}$ & $67 \mathrm{~b}$ & $84 \mathrm{a}$ \\
$\mathbf{0 7}$ & $87 \mathrm{~b}$ & $82 \mathrm{a}$ & $61 \mathrm{c}$ & $82 \mathrm{a}$ \\
$\mathbf{0 8}$ & $83 \mathrm{~b}$ & $80 \mathrm{a}$ & $56 \mathrm{c}$ & $78 \mathrm{~b}$ \\
$\mathbf{0 9}$ & $73 \mathrm{~d}$ & $67 \mathrm{~b}$ & $38 \mathrm{~d}$ & $63 \mathrm{~d}$ \\
$\mathbf{1 0}$ (Parte baixa) & $71 \mathrm{~d}$ & $64 \mathrm{c}$ & $37 \mathrm{~d}$ & $57 \mathrm{e}$ \\
$\mathbf{1 1}$ (Parte alta) & $90 \mathrm{a}$ & $89 \mathrm{a}$ & $71 \mathrm{a}$ & $80 \mathrm{~b}$ \\
$\mathbf{1 2}$ & $88 \mathrm{~b}$ & $85 \mathrm{a}$ & $67 \mathrm{~b}$ & $78 \mathrm{~b}$ \\
$\mathbf{1 3}$ & $88 \mathrm{~b}$ & $81 \mathrm{a}$ & $68 \mathrm{~b}$ & $78 \mathrm{~b}$ \\
$\mathbf{1 4}$ & $89 \mathrm{~b}$ & $86 \mathrm{a}$ & $67 \mathrm{~b}$ & $80 \mathrm{~b}$ \\
$\mathbf{1 5}$ & $87 \mathrm{~b}$ & $84 \mathrm{a}$ & $64 \mathrm{~b}$ & $77 \mathrm{~b}$ \\
$\mathbf{1 6}$ & $85 \mathrm{~b}$ & $71 \mathrm{~b}$ & $56 \mathrm{c}$ & $72 \mathrm{c}$ \\
$\mathbf{1 7}$ & $85 \mathrm{~b}$ & $71 \mathrm{~b}$ & $53 \mathrm{c}$ & $73 \mathrm{c}$ \\
$\mathbf{1 8}$ & $79 \mathrm{c}$ & $68 \mathrm{~b}$ & $43 \mathrm{~d}$ & $68 \mathrm{~d}$ \\
$\mathbf{1 9}$ & $76 \mathrm{c}$ & $59 \mathrm{c}$ & $32 \mathrm{e}$ & $58 \mathrm{e}$ \\
$\mathbf{2 0}$ (Parte baixa) & $68 \mathrm{~d}$ & $59 \mathrm{c}$ & $27 \mathrm{e}$ & $53 \mathrm{e}$ \\
\hline CV (\%) & 4,4 & 8,12 & 9,54 & 4,8 \\
\hline
\end{tabular}

Médias seguidas pela mesma letra não diferem entre si pelo teste de Scott-Knott em nível de $5 \%$ de probabilidade.

De maneira similar, o teste de envelhecimento acelerado possibilitou identificar três faixas de vigor, com superioridade nas frações da faixa de 1 a 5, vigor médio na fração 8 e na faixa de 11 a 15 e inferioridade nas faixas de 9 a 10 e de 16 a 20. Já Ferreira e Sá (2010), observaram que as várias etapas do beneficiamento são eficientes para melhorar a qualidade fisiológica de lotes de sementes.

De maneira geral verifica-se que a mesa de gravidade possibilitou a separação do lote em três níveis de qualidade fisiológica, destacando a parte alta da mesa com sementes de maior qualidade fisiológica, concordando com resultados obtidos por Deschamps (2005), também em sementes de soja.

\section{CONCLUSÕES}

As frações de maior qualidade física e fisiológica representam $80 \%$ do lote de sementes ao serem beneficiadas na mesa de gravidade;
A qualidade física e fisiológica da fração repasse na mesa de gravidade representa em média $10 \%$ do lote e permite aproveitamento de até $80 \%$.

\section{REFERÊNCIAS}

BRASIL. Ministério da Agricultura. Regras para análise de sementes. Brasília: Ministério da Agricultura, 2009, 365p.

BICCA, F. M. Qualidade de sementes de arroz beneficiadas em mesa de gravidade. 1996. $64 \mathrm{f}$. Dissertação (Mestrado) - Universidade Federal de Pelotas, Pelotas-RG, 1996.

BUITRAGO, I.C.; VILLELA, F.A.; TILLMANN, M.A.A.; SILVA, J.B. Perdas e qualidade de sementes de feijão beneficiados em máquina de ventilador e peneiras e mesa de gravidade. Revista Brasileira de Sementes, v.13, n.2, p.99-104, 1991. 
http://dx.doi.org/10.17801/0101-

3122/rbs.v13n2p99-104

DESCHAMPS, L.H. Qualidade de sementes de soja e de seu repasse beneficiados em mesa de gravidade. 2005. 36f. Dissertação (Mestrado) Universidade Federal de Pelotas, Pelotas-RG, 2005.

FERREIRA, R.L.; SÁ, M.E. Contribuição de etapas do beneficiamento na qualidade fisiológica de sementes de dois híbridos de milho. Revista Brasileira de Sementes, v.32, p.99-110, 2010. http://dx.doi.org/10.1590/S0101-

$\underline{31222010000400011}$

GAUL, A.D.; MISRA, M.K.; BERN, C.J.; HURBURGH, C.R. Variation of physical properties in gravity separated soybeans. Transactions of ASAE, v.29, n.4, p.1146-1149, 1986.

JUVINO, A. N.; RESENDE, O.; COSTA, L. M.; SALES, J.F. Vigor da cultivar BMX Potência RR de soja durante $O$ beneficiamento e períodos de armazenamento. Revista Brasileira de Engenharia Agricola e Ambiental-Agriambi, v.18, n.8, 2014. http://dx.doi.org/10.1590/18071929/agriambi.v18n08p844-850

KRZYZANOWSKI, F.; FRANÇA, J. B.; COSTA, N. P. Teste de hipoclorito de sódio para sementes de soja. Londrina: EMBRAPA, 2004. (Circular Técnica; 37)

MARCOS FILHO, J. Teste de envelhecimento acelerado. In: KRZYZANOWSKI, F.C.; VIEIRA, R.D.; FRANÇA-NETO, J.B. (Eds.). Vigor de sementes: conceitos e testes. Londrina: ABRATES, 1999. cap. 3, p.1-24.

MARCOS FILHO, J. Importância do potencial fisiológico da semente de soja. Informativo ABRATES, v.23, p.21-23, 2013.

PESKE, S. T.; BAUDET, L. Beneficiamento de Sementes. In: PESKE, S.T.; ROZENTHAL M.; ROTA, G.M. (Orgs.). Sementes: fundamentos científicos e tecnológicos. Pelotas: Universitária, 2003. v. 01, p. 323-367.

SILVA FILHO, P.M. Desempenho de plantas e sementes de soja classificadas por tamanho e densidade. 1994. 64f. Dissertação (Mestrado) -
Universidade Federal de Pelotas, Pelotas-RG, 1994.

TUNES, L.M.; PEDROSO, D.C.; BADINELLI, P.G.; TAVARES, L.C.; RUFINO, C.A.; BARROS, A.C.S.A.; MUNIZ, M.F.B. Envelhecimento acelerado em sementes de azevém com e sem solução salina e saturada. Ciência Rural, v.41, p.33- 37, 2011. http://dx.doi.org/10.1590/S0103$\underline{84782011000100006}$

Recebido para publicação em 15/09/2017 Revisado em 24/11/2017

Aceito em 25/01/2018 